Adv. Studies Theor. Phys., Vol. 7, 2013, no. 8, 363 - 366

HIKARI Ltd, www.m-hikari.com

\title{
GSAT with Adaptive Score Function
}

\author{
Vladimir Popov \\ Department of Intelligent Systems and Robotics \\ Ural Federal University \\ 620083 Ekaterinburg, Russia \\ Vladimir.Popov@usu.ru
}

Copyright (c) 2013 Vladimir Popov. This is an open access article distributed under the Creative Commons Attribution License, which permits unrestricted use, distribution, and reproduction in any medium, provided the original work is properly cited.

\begin{abstract}
GSAT is a well-known satisfiability search algorithm. In this paper we consider a modification of GSAT. In particular, we consider an adaptive score function.
\end{abstract}

PACS: $02.70 . \mathrm{Rr}$

Keywords: adaptive score function, GSAT, satisfiability

Investigation of efficient satisfiability algorithms and encoding different hard problems as instances of satisfiability has caused considerable interest recently (see e.g. [1, 2]). GSAT is a well-known satisfiability algorithm [3]. Note that GSAT applies only to clausal formulas. The notion of score plays a key role in GSAT. Although, some modification of the score function are considered, usually, the score function is the number of clauses of formula which are falsified by the truth assignment (see e.g. [4]). In this paper we consider a modification of the score function for clausal formulas with different types of clauses.

At first, we consider a general schema for standard GSAT (see Figure 1). We use the notation from [5]. Let $f(z[1], \ldots, z[n])$ be a CNF. Let $T$ be a truth assignment for the variables of $f$. The function $\operatorname{score}(T, f)$ is the number of clauses of $f$ which are falsified by T. GSAT performs an search for a satisfying truth assignment for the variables of $f$, starting from a random assignment provided by initial $(f)$. The successive assignment is obtained by inverting the truth value of one single variable $V$ in $T$. The value of $V$ is chosen to minimize 
the score. Let $T_{i}$ be the sequence obtained from $T$ by inverting the value of $i$ th variable $V_{i}$. The function hill $-\operatorname{climb}(f, T)$ returns the set Poss - flips of the variables $V_{r}$ which minimize $\operatorname{score}\left(T_{r}, f\right)$. The function pick(Poss - flips) chooses randomly one of elements of Poss - flips. The function $f l i p(V, T)$ returns $T$ with $V$ 's value inverted. The function $U$ pdateScores $(f, V)$ updates score.

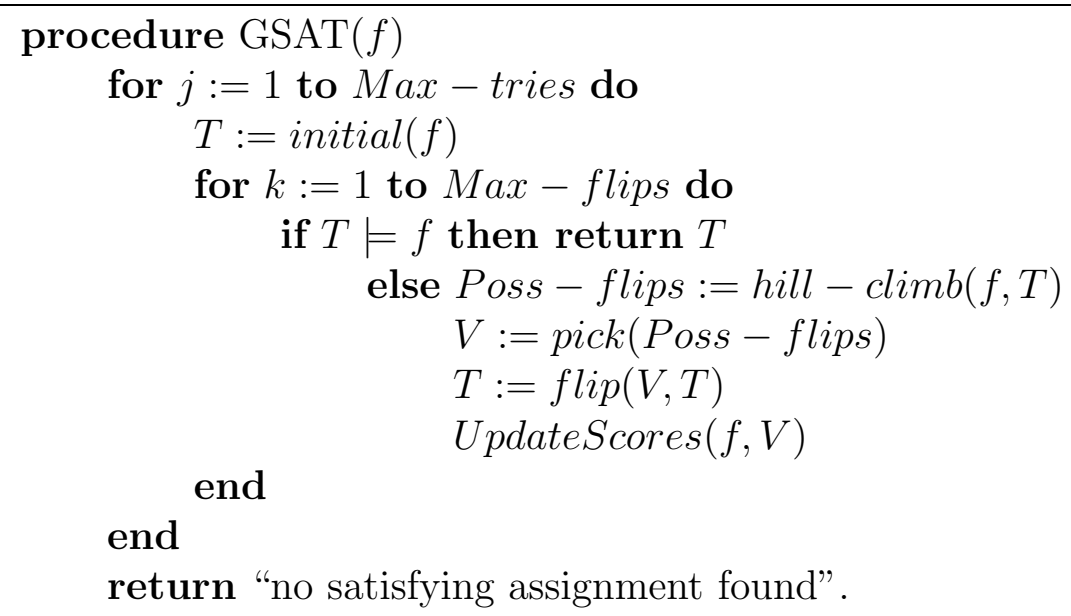

Figure 1: A general schema for GSAT.

The function $\operatorname{score}(T, f)$ is the number of clauses of $f$ which are falsified by $T$. In general, there are a number of different types of clauses. For instance, there is some difference between clauses $x$ and $y \vee z$. But, without additional infirmation, we can not give reasonable score of such difference. In particular, if $x=y=z=u=v=0$,

$$
\begin{aligned}
x & \wedge(y \vee z) \wedge(\neg x \vee u) \wedge(\neg x \vee v) \wedge(y \vee u), \\
x \wedge(y \vee z) & \wedge(\neg x \vee u) \wedge(\neg y \vee u) \wedge(\neg y \vee v) \wedge(\neg z \vee u) \wedge(\neg z \vee v),
\end{aligned}
$$

then we need modify two values of variables $(u$ and $v$ ) for $x$ and only one value of variable $(u)$ for $y \vee z$ in formula (1), but we need modify two values of variables $(u$ and $v$ ) for $y \vee z$ and only one value of variable $(u)$ for $x$ in formula (2). So, we can assume that the score of $x$ higher than the score of $y \vee z$, for formula (1), and the score of $x$ lower than the score of $y \vee z$, for formula (2).

Let $\#$ occ $(k, f, z[i])$ be the number of positive occurrences of $z[i]$ in clauses of type $x_{1} \vee \ldots \vee x_{k}, \#$ occ $(k, f, \neg z[i])$ be the number of occurrences of $\neg z[i]$ in clauses of type $x_{1} \vee \ldots \vee x_{k}$. Let $S(T, f)$ be the set of clauses of $f$ which are falsified by $T$. We assume that

$$
\operatorname{score}(T, f)=\sum_{x_{1} \vee \ldots \vee x_{p} \in S(T, f)} \sum_{1 \leq i \leq q, 1 \leq j \leq p}\left(\alpha\left(\# \text { occ }\left(i, f, x_{j}\right)\right)+\beta\left(\# \text { occ }\left(i, f, \neg x_{j}\right)\right)\right) .
$$


We use a genetic algorithm for prediction of values of $\alpha$ and $\beta$.

Now, we consider a special class of formulas 3-2-CNF. We assume that any clause of formula from this class belongs the set

$$
\{z[i] \vee z[j] \vee z[l], \neg z[i] \vee \neg z[j] \mid i, j, l \in N\}
$$

Theorem. For any 3-CNF $f$, there is a formula $g$ such that any clause of $g$ belongs the set (3) and $f$ is satisfiable if and only if $g$ is satisfiable.

Proof. It is easy to see that $x_{1} \vee x_{2} \vee \neg x_{3}$ is satisfiable if and only if $\left(x_{1} \vee x_{2} \vee x_{4}\right) \wedge\left(\neg x_{3} \vee \neg x_{4}\right)$ is satisfiable.

In view of theorem, we can be sure that the class $3-2-\mathrm{CNF}$ is sufficiently general. In our experiments, we consider GSAT with standard score function and GSAT with our score function (GSAT-ASF) for CNFs, 3-CNFs, and 3-2CNFs. Selected experimental results are given in Table 1.

\begin{tabular}{|l|lll|}
\hline & $3-\mathrm{CNF}$ & $3-2-\mathrm{CNF}$ & $\mathrm{CNF}$ \\
\hline GSAT & $86 \%$ & $81 \%$ & $79 \%$ \\
GSAT-ASF, $G=10^{4}$ & $91 \%$ & $89 \%$ & $81 \%$ \\
GSAT-ASF, $G=10^{5}$ & $92 \%$ & $94 \%$ & $85 \%$ \\
GSAT-ASF, $G=10^{6}$ & $93 \%$ & $95 \%$ & $88 \%$ \\
\hline
\end{tabular}

Table 1: A number of solved formulas for GSAT and GSAT-ASF where $G$ is a number of generations of genetic algorithm.

ACKNOWLEDGEMENTS. The work was partially supported by Analytical Departmental Program "Developing the scientific potential of high school" 8.1616.2011.

\section{References}

[1] A. Gorbenko and V. Popov, SAT Solvers for the Problem of Sensor Placement, Advanced Studies in Theoretical Physics, 6 (2012), 1235-1238.

[2] A. Gorbenko and V. Popov, Task-resource Scheduling Problem, International Journal of Automation and Computing, 9 (2012), 429-441.

[3] B. Selman, H. Levesque, and D. Mitchell, A new method for solving hard satisfiability problems, Proceedings of the Tenth National Conference on Artificial Intelligence, (1992), 440-446.

[4] R. Sebastiani, Applying GSAT to non-clausal formulas, Journal of Artificial Intelligence Research, 1 (1993), 309-314. 
[5] I.P. Gent and T. Walsh, Towards an Understanding of Hill-Climbing Procedures for SAT, Proceedings of the Eleventh National Conference on Artificial Intelligence, (1993), 28-33.

Received: February 12, 2013 